Pacific Journal of Mathematics

COUNTABLE ORDINALS AND THE ANALYTICAL 


\section{COUNTABLE ORDINALS AND THE ANALYTICAL HIERARCHY, I}

\section{A. S. KECHRIS}

The following results are proved, using the axiom of Projective Determinacy: (i) For $n \geqq 1$, every $\Pi_{2 n+1}^{1}$ set of countable ordinals contains a $\Delta_{2 n+1}^{1}$ ordinal, (ii) For $n \geqq 1$, the set of reals $\Delta_{2 n}^{\prime}$ in an ordinal is equal to the largest countable $\sum_{2 n}^{1}$ set and (iii) Every real is $\Delta_{n}^{1}$ inside some transitive model of set theory if and only if $n \geqq 4$.

In general we shall use the terminology and notation of [3]. In particular letters $i, j, k, \cdots$ are used as variables over $\omega=\{0,1,2, \cdots\}$ and $\alpha, \beta, \gamma, \cdots$ as variables over ${ }^{\omega} \omega(=$ the set of reals $)$. For a collection of sets of reals $\Gamma$, Determinacy $(\Gamma)$ abbreviates the statement that every set in $\Gamma$ is determined and Projective Determinacy (PD) is the axiom that every projective set is determined.

1. An ordinal basis theorem. A well known boundedness result in recursion theory asserts that if $W O=\left\{\alpha: \leqq{ }_{\alpha}\right.$ is a wellordering $\}$, where $\leqq_{\alpha}=\left\{(m, n): \alpha\left(2^{m} \cdot 3^{n}\right)=0\right\}$ and $A \subseteq W O$ is $\Sigma_{1}^{1}$, then $\sup \{|\alpha|: \alpha \in A\}<\delta_{1}^{1}$, where for $\alpha \in W O,|\alpha|=$ length $\left(\leqq_{\alpha}\right.$ ) and $\delta_{n}^{1}=\sup \left\{|\alpha|: \alpha \in \Delta_{n}^{1} \& \alpha \in W O\right\}$. We prove below a generalization of this fact to all odd levels of the analytical hierarchy.

THEOREM 1.1. Assume Projective Determinacy, when $n \geqq 1$. If $A \subseteq W O$ is $\sum_{2 n+1}^{1}$ and $\sup \{|\alpha|: \alpha \in A\}<\mathcal{N}_{1}$, then $\sup \{|\alpha|: \alpha \in A\}<$ $\delta_{2 n+1}^{1}$.

Proof. For notational simplicity let us take $n=1$ as a typical case. Thus let $A \subseteq W O$ be $\Sigma_{3}^{1}$ and assume $\sup \{|\alpha|: \alpha \in A\}<\boldsymbol{N}_{1}$. Let $B \subseteq{ }^{\omega} \omega$ be $\Pi_{2}^{1}$ and $f:{ }^{\omega} \omega \rightarrow{ }^{\omega} \omega$ recursive such that $f[B]=A$. Consider then the following game: Player I plays $\beta$, player II plays $\gamma$ and II wins iff $\gamma \in W O \&(\beta \in B \rightarrow|f(\beta)| \leqq|\gamma|)$. Clearly player II has a winning strategy in this game. But his payoff set is $\Sigma_{2}^{1}$, so by a result of Moschovakis [6] he has a winning strategy $\tau$ which is $\Delta_{3}^{1}$. Let $T=$ $\left\{\beta * \tau: \beta \in{ }^{\omega} \omega\right\}$, where $\beta * \tau$ is the result of II's moves following $\tau$ when I plays $\beta$. Then $T \subseteq W O$ and $T$ is $\Sigma_{1}^{1}(\tau)$, so by the Boundedness Theorem

$$
\sup \{|\gamma|: \gamma \in T\}<\delta_{1}^{1}(\tau)<\delta_{3}^{1}
$$


But clearly

$$
\sup \{|\alpha|: \alpha \in A\} \leqq \sup \{|\gamma|: \gamma \in T\}
$$

It is not hard now to reformulate Theorem 1.1. into a basis theorem. Call a set of ordinals $X \subseteq N_{1} \Pi_{n}^{1}$ if $\{\alpha \in W O:|\alpha| \in X\}$ is $\Pi_{n}^{1}$. Recall that a countable ordinal $\xi$ is called $\Delta_{n}^{1}$ iff $\xi<\delta_{n}^{1}$.

Theorem 1.2. Assume Projective Determinacy and $n \geqq 1$. Every nonempty $\Pi_{2 n+1}^{1}$ set of ordinals contains a $\Delta_{2 n+1}^{1}$ ordinal.

Proof. Take again $n=1$. Let $X \subseteq N_{1}$ be $\Pi_{3}^{1}$ and consider $A=$ $\{\alpha \in W O:|\alpha| \leqq \min X\}$. Then $\alpha \in A \Leftrightarrow \alpha \in W O \& \forall m\left(\left|\alpha_{m}\right| \notin X\right)$, where $\alpha_{m}$ is a real coding the restriction of $\leqq_{\alpha}$ to its initial segment determined by $m$. Clearly $A$ is $\Sigma_{3}^{1}$ and $A \subseteq W O$, so by Theorem 1.1. $\sup \{|\alpha|: \alpha \in A\}=\min X<\delta_{3}^{1}$, thus $X$ contains a $\Delta_{3}^{1}$ ordinal.

Remark. If $X=\left\{\xi<\aleph_{1}: \omega_{0}<\xi\right.$ is admissible $\}$ then $X$ is $\Pi_{1}^{1}$ but contains no $\Delta_{1}^{1}$ ordinal.

2. The set of reals $\Delta_{n}^{1}$ in an ordinal. A real $\alpha$ is called $\Delta_{n}^{1}$ in an ordinal $\xi<\aleph_{1}$ if $\alpha$ is $\Delta_{n}^{1}$ in every real $\beta \in W O$ such that $|\beta|=\xi$. A simple argument shows that a real is $\Delta_{1}^{1}$ in an ordinal iff it is $\Delta_{2}^{1}$ in an ordinal iff it is constructible. Martin and Solovay [4] proved that under PD, the set of reals $\Delta_{2 n+1}^{1}$ in an ordinal, when $n \geqq 1$, is exactly the set $Q_{2 n+1}$ (see [4] or [3].) [The set $Q_{2 n+1}$ can be defined in many equivalent ways. One of the most suggestive ones is the following: $Q_{2 n+1}=\left\{\alpha: \alpha\right.$ belongs to every model of $Z F C+P D$ for which $\Sigma_{2 n}^{1}$ formulas are absolute\}]. We shall identify below the set of reals which are $\Delta_{2 n}^{1}$ in an ordinal. Before doing this though we shall give as an application of Theorem 1.1. a new proof of the result of Martin and Solovay. Their original proof used forcing.

Theorem 2.1. (Martin-Solovay [4]). Assume Projective Determinacy and $n \geqq 1$. Then $Q_{2 n+1}=\left\{\alpha: \alpha\right.$ is $\Delta_{2 n+1}^{1}$ in an ordinal $\}$.

Proof. Let $n=1$ again. From the results of [3] (especially the Lemma before Theorem (3B-3)) we can easily see that every real in $Q_{3}$ is $\Delta_{3}^{1}$ in an ordinal. Conversely, if $\alpha$ is $\Delta_{3}^{1}$ in an ordinal, then by Theorem $1.2, \alpha$ is $\Delta_{3}^{1}$ in an ordinal $\xi$ which is $\Delta_{3}^{1}$ in $\alpha$. Thus $A=\left\{\alpha: \alpha\right.$ is $\Delta_{3}^{1}$ in an ordinal $\}$ is $\Pi_{3}^{1}$. By Theorem (3B-3) of [3] the set $Q_{3}$ is characterized as the largest $\Pi_{3}^{1}$-bounded set, where a set $B \subseteq \omega^{\omega}$ is $\Pi_{3}^{1}$-bounded if for all predicates $P(\alpha, \beta)$ in $\Pi_{3}^{1}$ the predicate $\exists \alpha \in B P(\alpha, \beta)$ is also $\Pi_{3}^{1}$. To complete the proof it is thus enough to show that $A$ is $\Pi_{3}^{1}$ bounded. For any $\Pi_{3}^{1}$ predicate $P(\alpha, \beta)$ put 


$$
X_{\beta}=\left\{\xi<\mathcal{N}_{1}: \forall \gamma\left(\gamma \in W O \&|\gamma|=\xi \rightarrow \exists \alpha \in \Delta_{3}^{1}(\gamma)[P(\alpha, \beta) \& a \in A] .\right\}\right.
$$

Then $X_{\beta}$ is $\Pi_{3}^{1}$ in $\beta$ and $\exists \alpha \in A P(\alpha, \beta) \Rightarrow X_{\beta} \neq \varnothing$, so $\exists \alpha \in A P(\alpha, \beta) \Rightarrow$ $X_{\beta}$ contains an ordinal $\Delta_{3}^{1}$ in $\beta \Rightarrow \exists \alpha \in \Delta_{3}^{1}(\beta)[P(\alpha, \beta) \& \alpha \in A]$. Thus $\exists \alpha \in A P(\alpha, \beta) \Leftrightarrow \exists \alpha \in \Delta_{3}^{1}(\beta)[\alpha \in A \& P(\alpha, \beta)]$ and we are done.

We now proceed to identify the set of reals $\Delta_{2 n}^{1}$ in an ordinal. Our result generalizes the fact that $\left\{\alpha: \alpha\right.$ is $\Delta_{2}^{1}$ in an ordinal $\}=\{\alpha: \alpha$ is constructible $\}=C_{2}={ }^{\text {def }}$ the largest countable $\Sigma_{2}^{1}$ set of reals (we assume here that there are only countably many constructible reals).

Theorem 2.2. Assume Projective Determinacy and let $C_{2 n}=$ the largest countable $\Sigma_{2 n}^{1}$ set of reals. Then $C_{2 n}=\left\{\alpha: \alpha\right.$ is $\Delta_{2 n}^{1}$ in an ordinal\}.

Proof. For notational simplicity take $n=2$. By Theorem (1C-3) of [3], $C_{4} \subseteq\left\{\alpha: \alpha\right.$ is $\Delta_{4}^{1}$ in an ordinal $\}={ }^{\text {def }} S$. Since $S$ is countable it is enough to prove that $S$ is $\Sigma_{4}^{1}$.

For each countable ordinal $\xi \geqq \omega$ consider the space ${ }^{\omega} \xi$ with the product topology, where $\xi$ has the discrete topology. If $f \in{ }^{\omega} \xi$ let $\leqq_{f}$ be the relation on $\omega$ given by $m \leqq n \Leftrightarrow f(m) \leqq f(n)$. Write $\alpha \in \Delta_{4}^{\prime}(f)$ iff $\alpha \in \Delta_{4}^{1}\left(\leqq_{f}\right)$. We shall prove that if

$$
P=\left\{\alpha:(\exists \xi)\left(\omega \leqq \xi<\aleph_{1} \&\left\{f \in{ }^{\omega} \xi: \alpha \in \Delta_{4}^{\prime}(f)\right\} \text { is not meager }\right)\right\},
$$

then $P \in \Sigma_{4}^{1}$ and $P=S$. Note first that

$$
\alpha \in P \Leftrightarrow(\exists \sigma)\left(\sigma \in W O^{*} \&\left\{f \in{ }^{\omega}|\sigma|: \alpha \in \Delta_{4}^{\prime}(f)\right\} \text { is not meager }\right) \text {, }
$$

where $\alpha \in W O^{*} \Leftrightarrow \alpha \in W O \&|\alpha| \geqq \omega \& \leqq{ }_{\alpha}$ has field $\omega$. If $\sigma \in W O^{*}$ let $h_{\sigma}: \omega \rightarrow|\sigma|$ be the bijection such that $m \leqq{ }_{\sigma} n \Leftrightarrow h_{\sigma}(m) \leqq h_{\sigma}(n)$ and let $h_{\sigma}^{*}:{ }^{\omega} \omega \rightarrow{ }^{\omega}|\sigma|$ be given by $h_{\sigma}^{*}(\alpha)=h_{\sigma}{ }^{\circ} \alpha$. Then $h_{\sigma}^{*}$ is a homeomorphism of ${ }^{\omega} \omega$ with ${ }^{\omega}|\sigma|$. Thus

$$
\begin{aligned}
\alpha \in \mathrm{P} \Leftrightarrow(\exists \sigma)\left(\sigma \in \mathrm{WO}^{*} \& \mathrm{~h}_{\sigma}^{*-1}\left[\left\{\mathrm{f} \in{ }^{\omega}|\sigma|: \alpha \in \Delta_{4}^{1}(\mathrm{f})\right\}\right]\right. \\
\text { is not meager }) \\
\Leftrightarrow(\exists \sigma)\left(\sigma \in W O^{*} \&\left\{\beta: \alpha \in \Delta_{4}^{1}\left(h_{\sigma} \circ \beta\right)\right\}\right. \\
\text { is not meager }) \\
\Leftrightarrow(\exists \sigma)\left(\sigma \in W O^{*} \&\left\{\beta: \alpha \in \Delta_{4}^{1}\left(\leqq_{h_{\sigma} \beta}\right)\right\}\right. \\
\text { is not meager }) .
\end{aligned}
$$


But for $\sigma \in W O^{*}$,

$$
\begin{aligned}
m \leqq_{h_{\sigma} \beta} n & \Leftrightarrow h_{\sigma}(\beta(m)) \leqq h_{\sigma}(\beta(n)) \\
& \Leftrightarrow \beta(m) \leqq{ }_{\sigma} \beta(n) \\
& \Leftrightarrow H(\beta, \sigma)\left(2^{m} \cdot 3^{n}\right)=0
\end{aligned}
$$

where $H:{ }^{\omega} \omega \times{ }^{\omega} \omega \rightarrow{ }^{\omega} 2$ is recursive and $H(\beta, \sigma)(k)=1$, if $\forall m \forall n\left(k \neq 2^{m} \cdot 3^{n}\right)$. So

$$
\alpha \in P \Leftrightarrow(\exists \sigma)\left(\sigma \in W O^{*} \&\left\{\beta: \alpha \in \Delta_{4}^{1}(H(\beta, \sigma))\right\} \text { is not meager }\right)
$$

which by Theorem $2.2 .5(\mathrm{~b})$ of [2] shows that $P$ is $\Sigma_{4}^{1}$.

We prove now that $S=P$. Clearly $S \subseteq P$. For the converse let $\alpha \in P$ and find $\xi \geqq \omega$ such that $\left\{f \in{ }^{\omega} \xi: \alpha \in \Delta_{4}^{1}(f)\right\}$ is not meager. Let $\sigma \in W O$ be such that $|\sigma|=\xi$. We shall show that $\alpha \in$ $\Delta_{4}^{1}(\sigma)$. Without loss of generality we can assume that $\leqq_{\sigma}$ has field all of $\omega$ (i.e., $\left.\sigma \in W O^{*}\right)$. Then as before $\left\{\beta: \alpha \in \Delta_{4}^{1}(H(\sigma, \beta))\right\}$ is not meager, thus $\left\{\beta: \alpha \in \Delta_{4}^{1}(\sigma, \beta)\right\}$ is not meager. But then by Theorem 3.1.2.(b) of [2], $\alpha \in \Delta_{4}^{1}(\sigma)$.

3. Ordinals and reals $\Delta_{n}^{1}$ in models of set theory. Call a countable ordinal $\xi$ almost $\Delta_{n}^{1}$ if there is a countable transitive model $M$ of $Z F C$ such that $\xi \in M$ and $M \mid=\xi$ is $\Delta_{n}^{1}$. For $n=1,2$ it is trivial to see that $\xi$ is almost $\Delta_{n}^{1}$ iff $\xi$ is $\Delta_{n}^{1}$. By using a simple Solovay type game one can prove easily the following. (We call a transitive model $M$ of ZFC $\sum_{n}^{1}$-correct if $\sum_{n}^{1}$ formulas are absolute for $M$.)

Proposition 3.1. Assume Projective Determinacy. For any $n \geqq$ 1 and any countable ordinal $\xi$ there is a model $M$ of $Z F C+$ Determinacy $\left(\Delta_{2 n}^{1}\right)$ which is $\sum_{2 n}^{1}$-correct, $\xi \in M$ and $M \mid=\xi$ is $\Delta_{2 n+1}^{1}$.

Call now a real $\alpha$ almost $\Delta_{n}^{1}$ if there is a transitive countable model $M$ of $Z F C$ such that $\alpha \in M$ and $M \mid=\alpha$ is $\Delta_{n}^{1}$. Abbreviate by $D_{n}$ the statement $\forall \alpha\left(\alpha\right.$ is almost $\left.\Delta_{n}^{1}\right)$. As with ordinals, $\alpha$ is almost $\Delta_{n}^{1}$ iff $\alpha$ is $\Delta_{n}^{1}$, for $n=1,2$, so $D_{1}, D_{2}$ fail. Unlike the case of the ordinals $D_{3}$ also fails.

Theorem 3.2. Assume Projective Determinacy and let $D_{n} \Leftrightarrow$ $\forall \alpha\left(\alpha\right.$ is almost $\left.\Delta_{n}^{1}\right)$. Then $D_{n}$ holds iff $n \geqq 4$.

Proof. Clearly $\left\{\alpha: \alpha\right.$ is not almost $\left.\Delta_{n}^{1}\right\}$ is a $\Pi_{2}^{1}$ set, so if not empty it has a $\Delta_{4}^{1}$ solution, a contradiction if $n \geqq 4$. To complete the proof we show that $D_{3}$ fails. Let $\alpha_{0}$ be a real which codes a countable transitive 
model $N$ of $Z F C+$ Determinacy $\left(\Delta_{2}^{1}\right)$ which is $\Sigma_{2}^{1}$-correct. We show that $\alpha_{0}$ is not almost $\Delta_{3}^{1}$. If not, let $M$ be a countable transitive model of $Z F C$ such that $\alpha_{0} \in M$ and $M \mid=\alpha_{0}$ is $\Delta_{3}^{1}$. Then $N \in M$ and $\Sigma_{2}^{1}$ formulas are absolute from $M$ to $N$, so, since $N \mid=$ Determinacy $\left(\Delta_{2}^{1}\right)$, clearly $M \mid=$ Determinacy $\left(\Delta_{2}^{\prime}\right) \quad$ (in general we cannot conclude that $M \mid=$ Determinacy $\left.\left(\Delta_{2}^{1}\right)\right)$. By the arguments in [1] or [5] the class of $\Pi_{3}^{1}$ relations on $\omega$ has the prewellordering property, in $M$. But then by Moschovakis [7], $M \mid=$ "There is a $\Delta_{2}^{\prime}$ game in which I has a winning strategy and $\alpha_{0}$ is recursive in every strategy of this game". Since $N \mid=$ Determinacy $\left(\Delta_{2}^{1}\right)$ at least one of these strategies is in $N$, a contradiction.

Theorem 3.2 exposes a weak phenomenon which for the first time happens in the fourth level of the analytical hierarchy. Hopefully the discovery of more and especially stronger such phenomena will result in the understanding of the structural differences between the third and the fifth level of the analytical hierarchy. For that purpose it seems that what is needed is a deeper understanding of the role of uncountable ordinals and models of set theory in the analytical hierarchy.

\section{REFERENCES}

1. J. W. Addison and Y. N Moschovakis, Some consequences of the axiom of definable determinateness, Proc. Nat. Acad. Sci., USA 59 (1968), 708-712.

2. A. S. Kechris, Measure and category in effective descriptive set theory, Annals of Math. Logic, 5 (1973), 337-384.

3. - The theory of countable analytical sets, Trans. of Amer. Math. Soc., 202 (1975), 259-297.

4. D. A. Martin and R. M. Solovay, Basis theorems for $\Pi_{2 k}^{1}$ sets of reals, to appear.

5. D. A. Martin, The axiom of determinateness and reduction principles in the analytical hierarchy, Bull. Amer. Math. Soc., 74 (1968), 687-689.

6. Y. N. Moschovakis, Analytical definability in a playful universe, P. Suppes et al., eds. Logic, Methodology and Philosophy of Science IV, North Holland, 1973, 77-85.

7. _ - Proof of a conjecture of Martin, mimeographed notes.

Received August 29, 1974. Research partially supported by NSF grant GP 27964.

MassachusetTs Institute of Technology

AND

California Institute of Technology 

D. E. Bennett, Strongly unicoherent continua ............................. 1

Walter R. Bloom, Sets of p-spectral synthesis ................................ 7

R. T. Bumby and D. E. Dobbs, Amitsur cohomology of quadratic extensions: Formulas and number-theoretic examples ................. 21

W. W. Comfort, Compactness-like properties for generalized weak topological sums

D. R. Dunninger and J. Locker, Monotone operators and nonlinear biharmonic boundary value problems ...

T. S. Erickson, W. S. Martindale, 3rd and J. M. Osborn, Prime nonassociative algebras

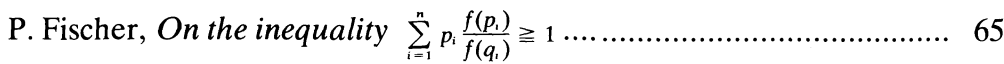

G. Fox and P. Morales, Compact subsets of a Tychonoff set ............... 75

R. Gilmer and J. F. Hoffmann, A characterization of Prüfer domains in terms of polynomials ......................................................... 81

L. C. Glaser, On tame Cantor sets in spheres having the same projection in each direction ......................................................... 87

Z. Goseki, On semigroups in which $X=X Y X=X Z X$ if and only if

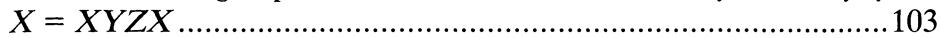

E. Grosswald, Rational valued series of exponentials and divisor

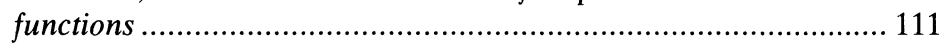

D. Handelman, Strongly semiprime rings ...................................... 115

J. N. Henry and D. C. Taylor, The $\bar{\beta}$ topology for $w^{*}$-algebras ............. 123

M. J. Hodel, Enumeration of weighted p-line arrays .......................... 141

S. K. Jain and S. Singh, Rings with quasiprojective left ideals .............. 169

S. Jeyaratnam, The diophantine equation $Y(Y+m)(Y+2 m) \times$

$$
(Y+3 m)=2 X(X+m)(X+2 m)(X+3 m) \ldots \ldots \ldots \ldots \ldots \ldots \ldots . . .183
$$

$\mathrm{R}$. Kane, On loop spaces without $p$ torsion .........................................189

Alvin J. Kay, Nonlinear integral equations and product integrals ..........203

A. S. Kechris, Countable ordinals and the analytic hierarchy, I ...........223

Ka-Sing Lau, A representation theorem for isometries of $C(X, E) \ldots \ldots . .229$

I. Madsen, On the action of the Dyer-Lashof algebra in $H_{*}(G)$..........235

R. C. Metzler, Positive linear functions, integration, and Choquet's theorem ........................................................................................ 277

A. Nobile, Some properties of the Nash blowing-up ............................297

G. E. Petersen and G. V. Welland, Plessner's theorem for Riesz conjugates 


\section{Pacific Journal of Mathematics}

\section{Vol. 60, No. $1 \quad$ September, 1975}

Donald Earl Bennett, Strongly unicoherent continua ................ 1

Walter Russell Bloom, Sets of p-spectral synthesis ................ 7

Richard Thomas Bumby and David Earl Dobbs, Amitsur cohomology of

quadratic extensions: formulas and number-theoretic examples .......

W. Wistar (William) Comfort, Compactness-like properties for generalized

weak topological sums ...................................

Dennis Robert Dunninger and John Stewart Locker, Monotone operators

and nonlinear biharmonic boundary value problems ..............

Theodore Erickson, Wallace Smith Martindale, III and J. Marshall Osborn,

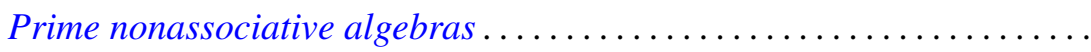

Pál Fischer, On the inequality $\sum_{i=0}^{n}\left[f\left(p_{i}\right) / f\left(q_{i}\right)\right] p_{i} \geq i \ldots \ldots \ldots \ldots \ldots$

Geoffrey Fox and Pedro Morales, Compact subsets of a Tychonoff set.......

Robert William Gilmer, Jr. and Joseph F. Hoffmann, A characterization of

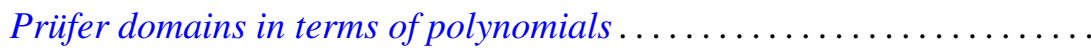

Leslie C. Glaser, On tame Cantor sets in spheres having the same projection

in each direction . ...................................

Zensiro Goseki, On semigroups in which $x=x y x=x z x$ if and only if

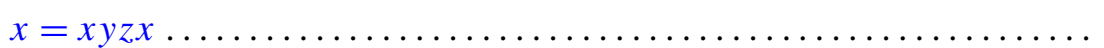

Emil Grosswald, Rational valued series of exponentials and divisor

functions.

David E. Handelman, Strongly semiprime rings

Jackson Neal Henry and Donald Curtis Taylor, The $\bar{\beta}$ topology for

$W^{*}$-algebras

Margaret Jones Hodel, Enumeration of weighted p-line arrays ...

Surender Kumar Jain and Surjeet Singh, Rings with quasi-projective left

ideals.

S. Jeyaratnam, The Diophantine equation

$$
Y(Y+m)(Y+2 m)(Y+3 m)=2 X(X+m)(X+2 m)(X+3 m) \ldots
$$

Richard Michael Kane, On loop spaces without $p$ torsion

Alvin John Kay, Nonlinear integral equations and product integrals ...

Alexander S. Kechris, Countable ordinals and the analytical hierarchy.

$I$.

Ka-Sing Lau, A representation theorem for isometries of $C(X, E)$

Ib Henning Madsen, On the action of the Dyer-Lashof algebra in $H_{*}(G)$

Richard C. Metzler, Positive linear functions, integration, and Choquet's

theorem.

Augusto Nobile, Some properties of the Nash blowing-up

Gerald E. Peterson and Grant Welland, Plessner's theorem for Riesz. 